\title{
Dimeric Copper and Lithium Thiolates: Comparison of Copper Thiolates with Their Lithium Congeners
}

\author{
Wenxing Zou, Qihao Zhu, James C. Fettinger and Philip P. Power* \\ Department of Chemistry, University of California, One Shields Avenue, Davis, California 95616, United States \\ Philip P. Power: pppower@ucdavis.edu \\ Supporting information
}

Table of Contents:

1. Characterization data for complex 1 S3

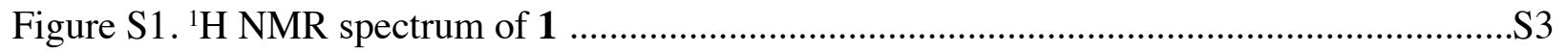

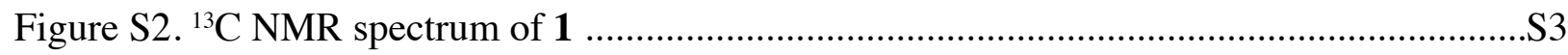

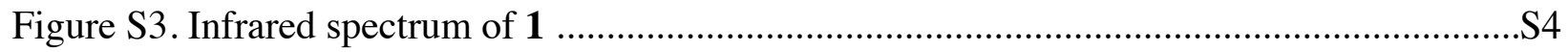

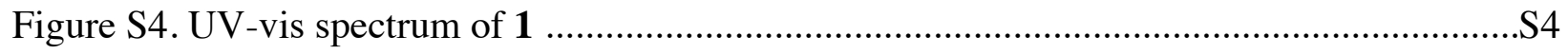

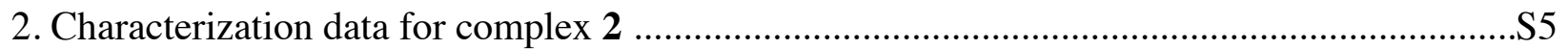

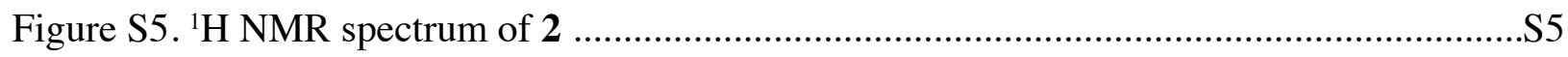

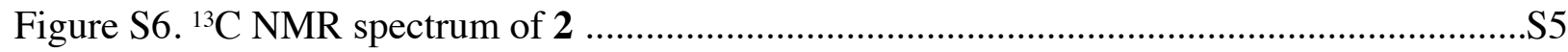

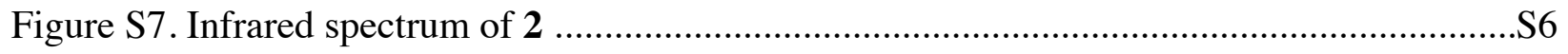

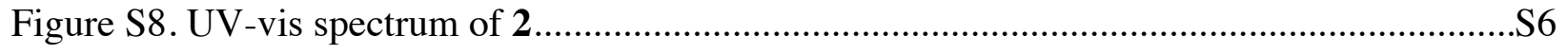

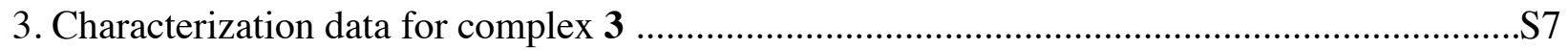




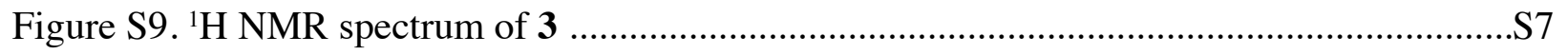

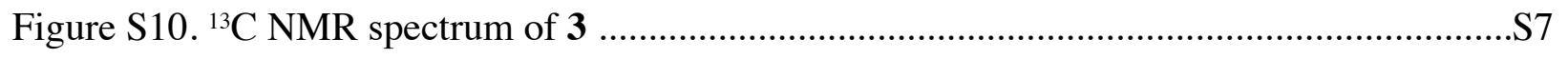

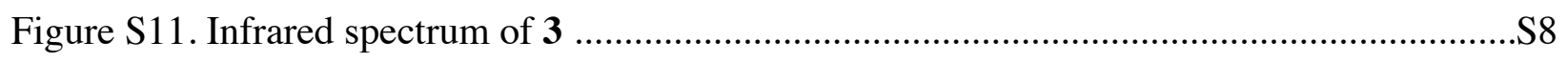

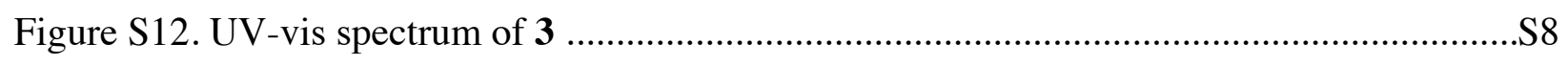

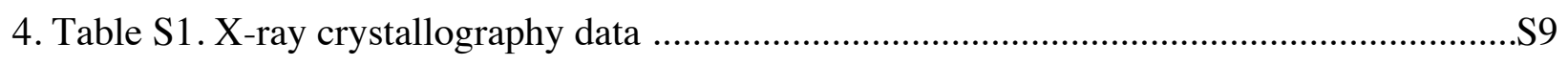

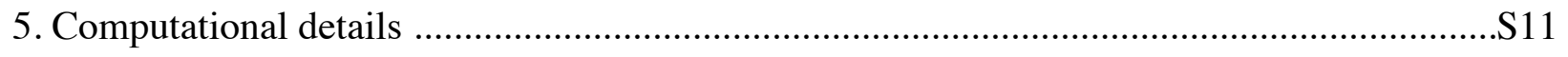

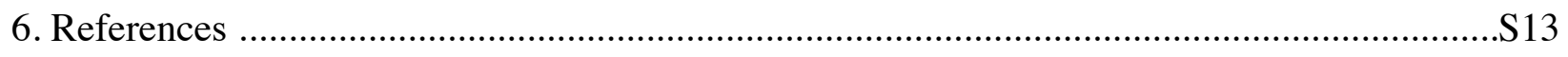




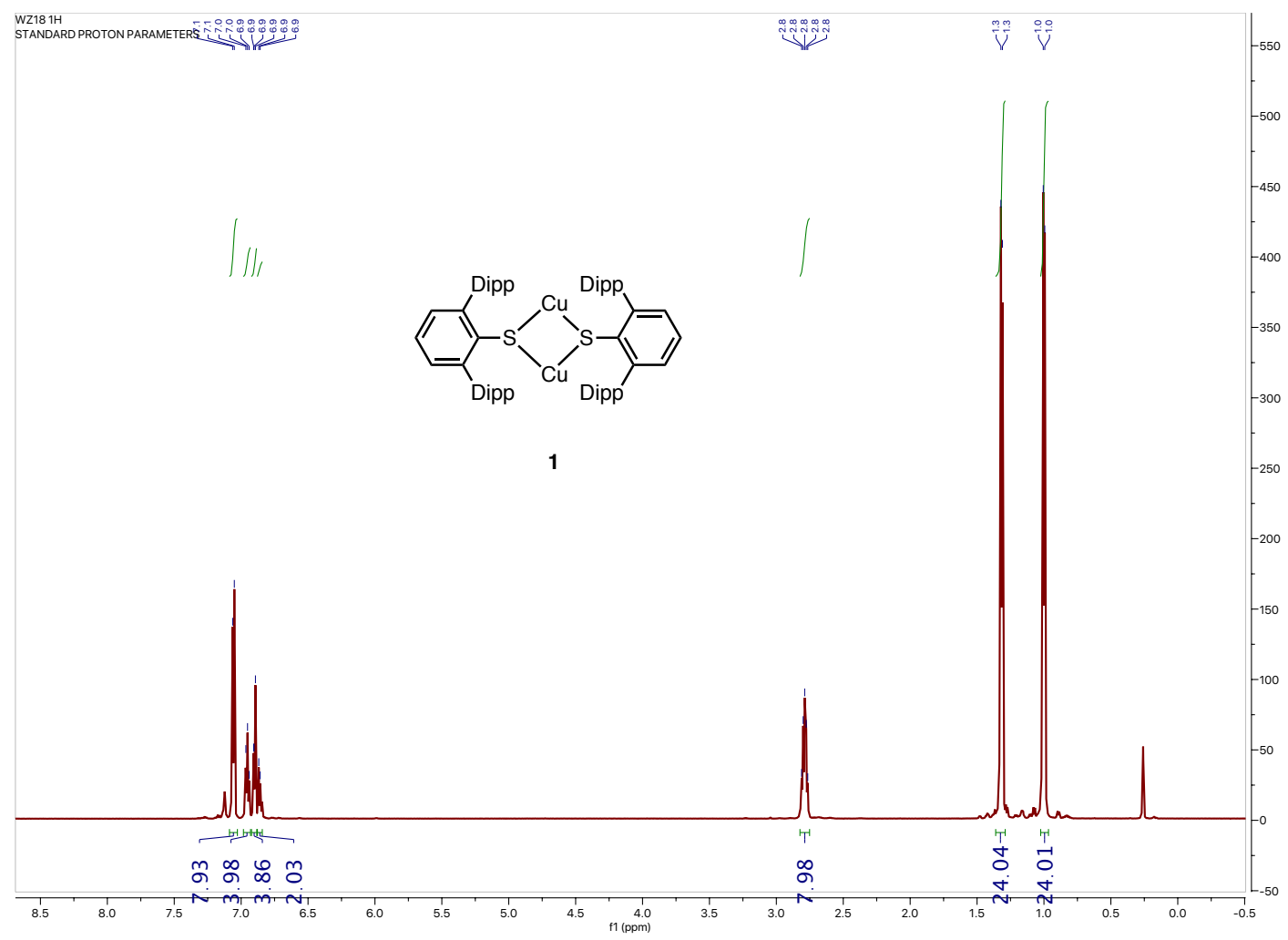

Figure S1. ${ }^{1} \mathrm{H}$ NMR spectrum of $\left\{\mathrm{CuSAr}^{\mathrm{iPr} 4}\right\}_{2}(\mathbf{1})\left(600 \mathrm{MHz}, \mathrm{C}_{6} \mathrm{D}_{6}, 298 \mathrm{~K}\right)$

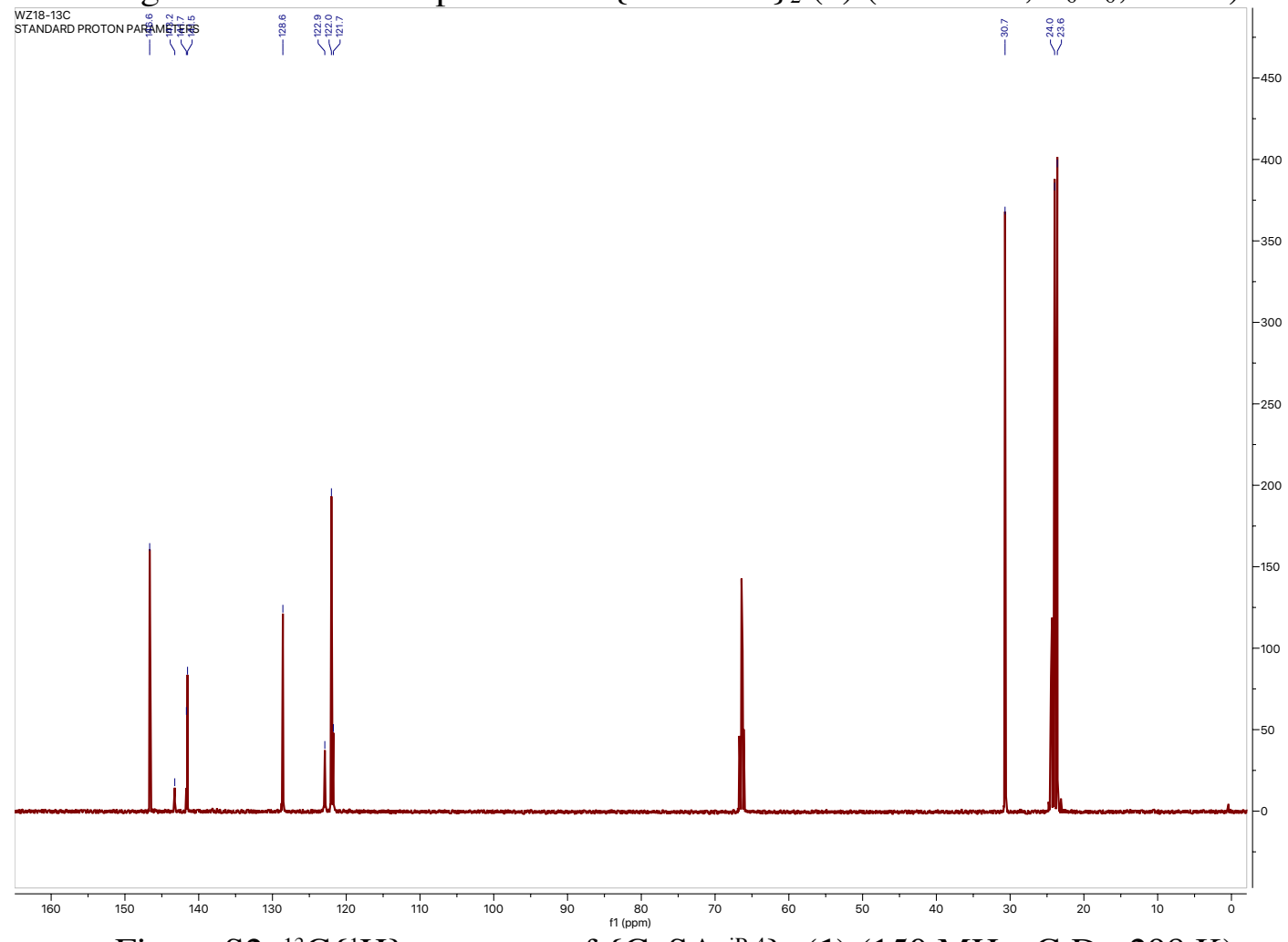

Figure S2. ${ }^{13} \mathrm{C}\left\{{ }^{1} \mathrm{H}\right\}$ spectrum of $\left\{\mathrm{CuSAr}^{\mathrm{iPr} 4}\right\}_{2}(\mathbf{1})\left(150 \mathrm{MHz}, \mathrm{C}_{6} \mathrm{D}_{6}, 298 \mathrm{~K}\right)$ 


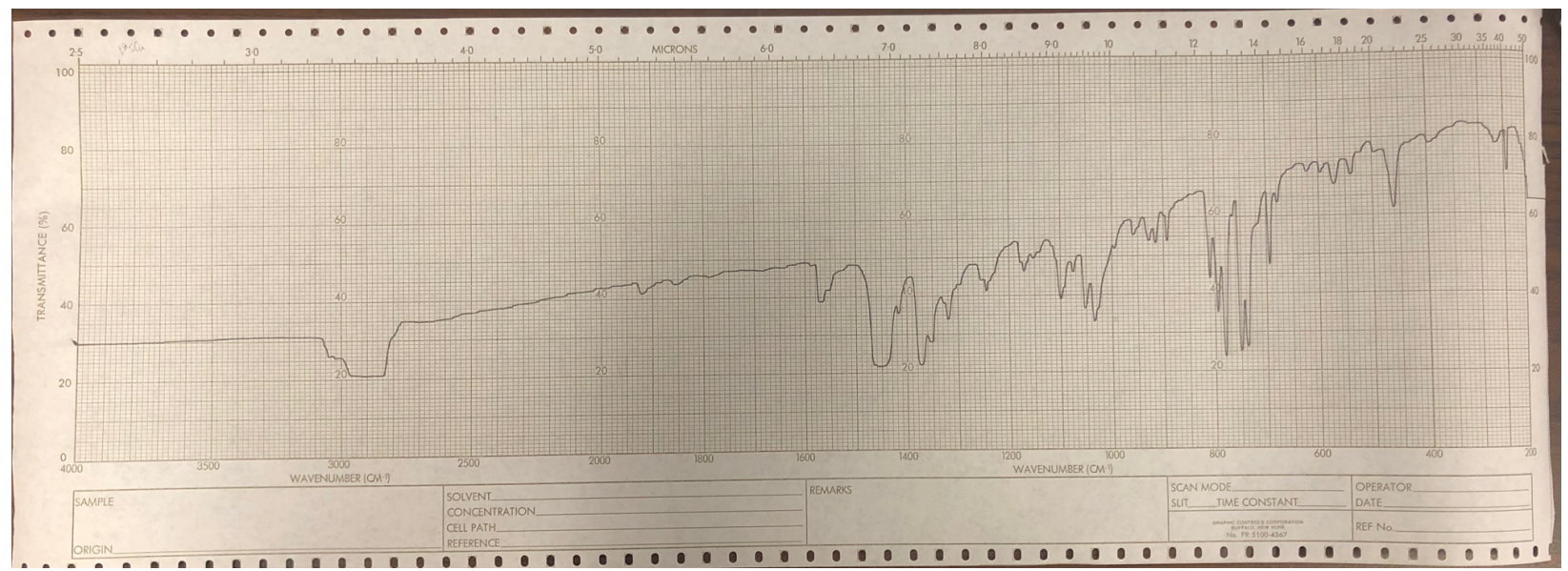

Figure S3. Infrared spectrum as a Nujol mull of $\left\{\mathrm{CuSAr}^{\mathrm{iPr} 4}\right\}_{2}(\mathbf{1})$ at $25^{\circ} \mathrm{C}$

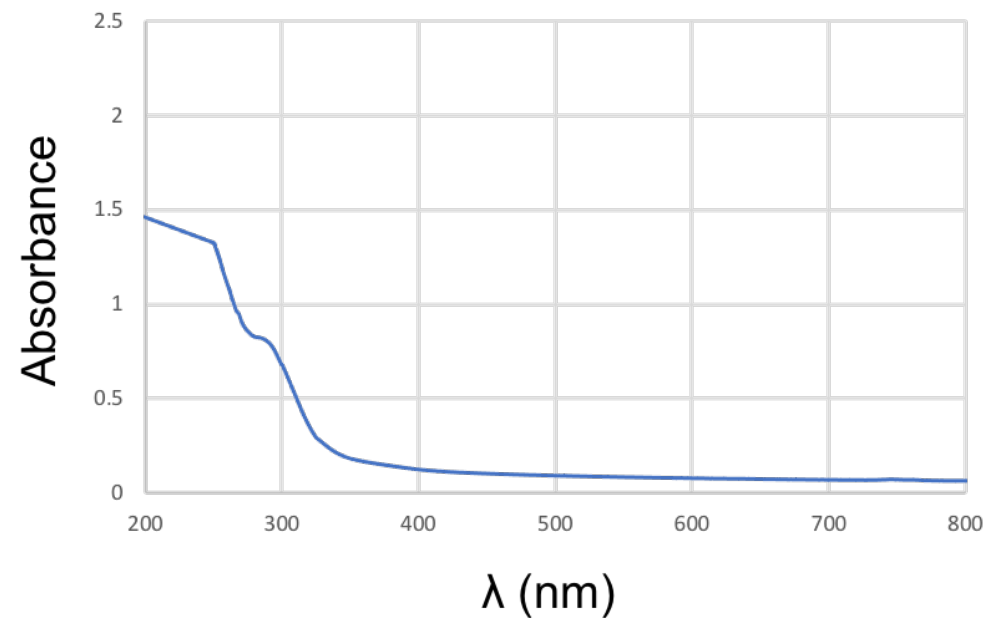

Figure S4. UV-Vis spectrum of $\left\{\mathrm{CuSAr}^{\mathrm{iPr} 4}\right\}_{2}(\mathbf{1})$ at $25^{\circ} \mathrm{C}(470 \mu \mathrm{M}$ in hexanes) 


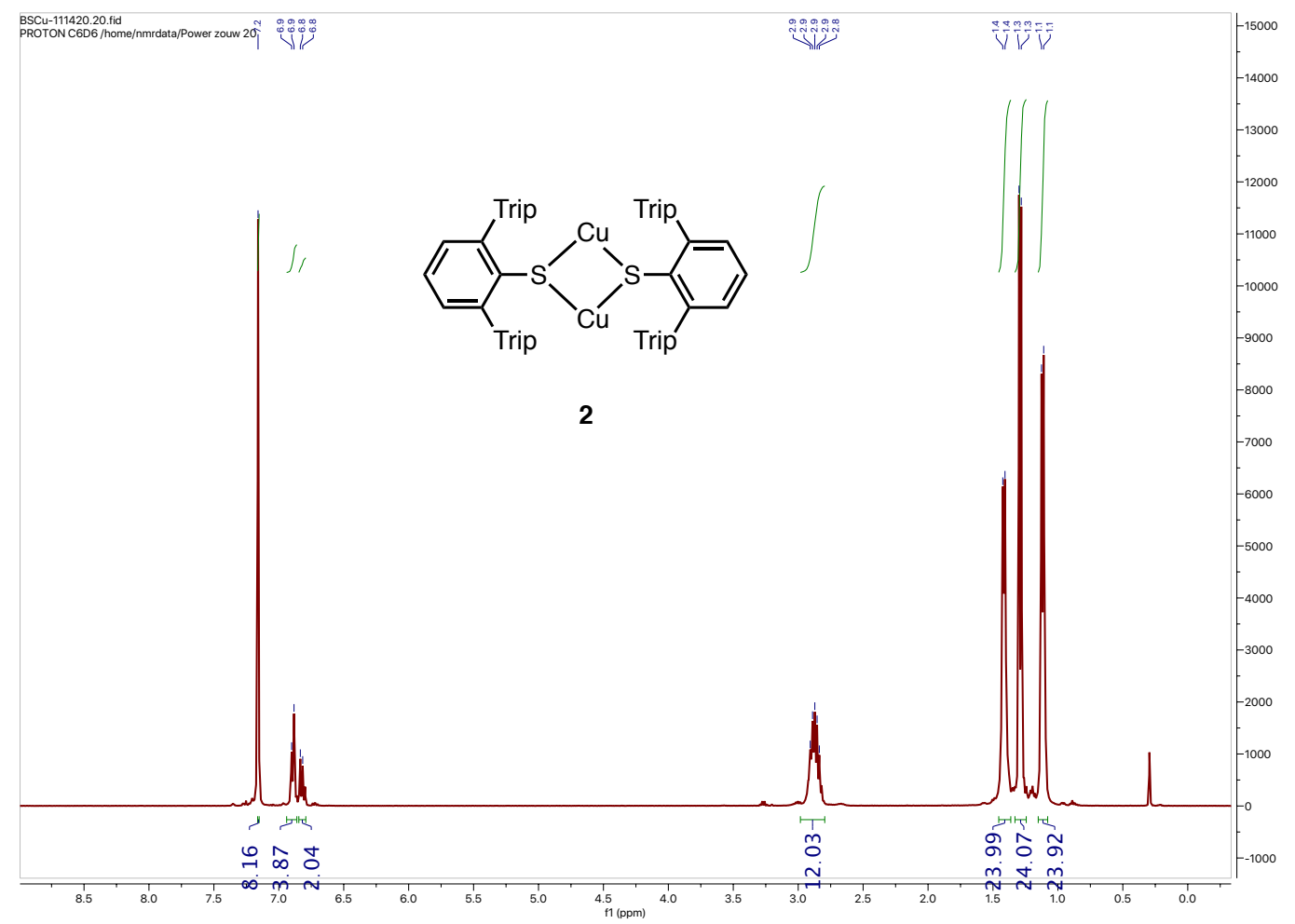

Figure S5. ${ }^{1} \mathrm{H}$ NMR spectrum of $\left\{\mathrm{CuSAr}^{\mathrm{Pr} 6}\right\}_{2}(2)\left(600 \mathrm{MHz}, \mathrm{C}_{6} \mathrm{D}_{6}, 298 \mathrm{~K}\right)$

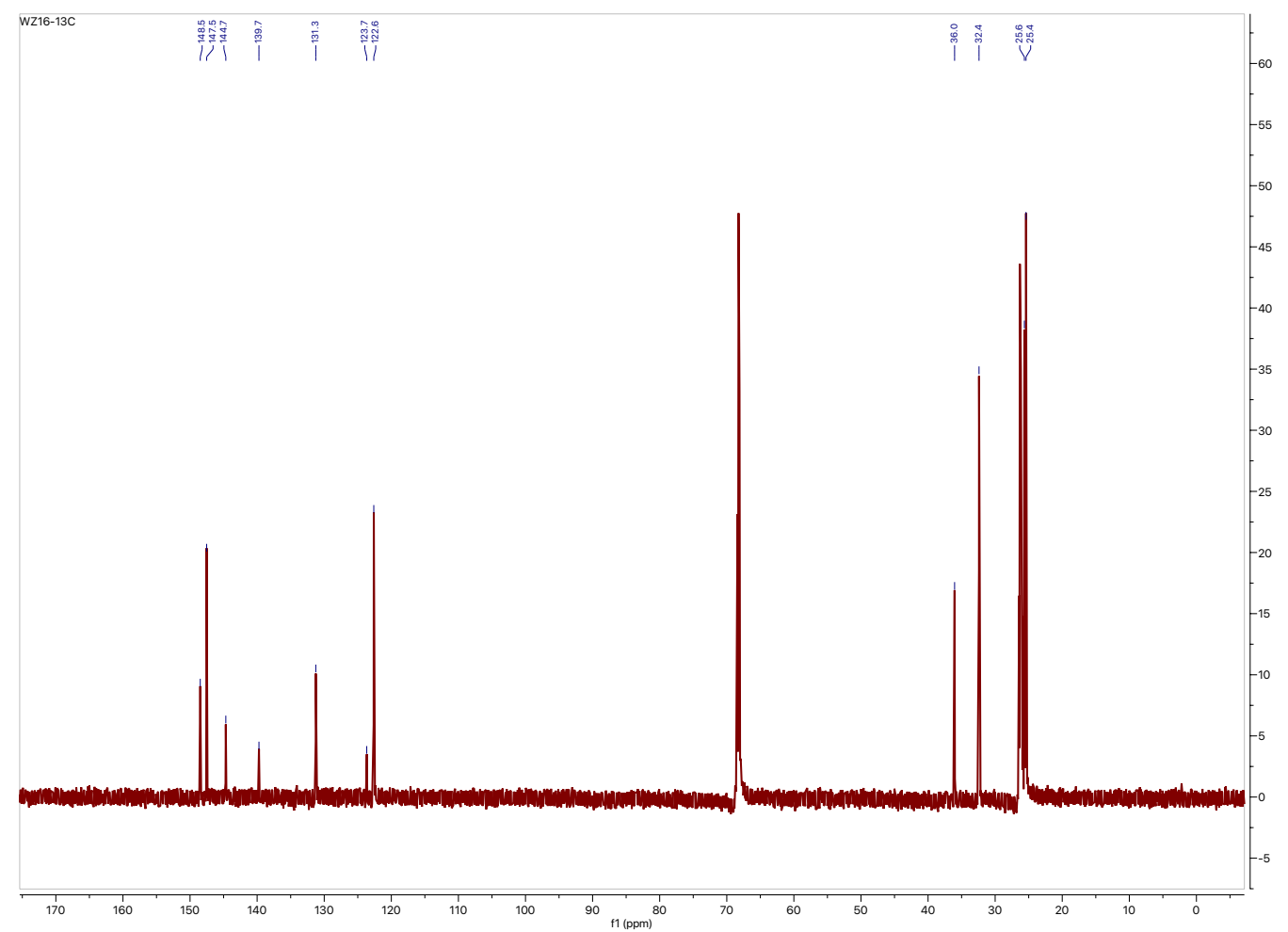

Figure S6. ${ }^{13} \mathrm{C}\left\{{ }^{1} \mathrm{H}\right\}$ NMR spectrum of $\left\{\mathrm{CuSAr}^{\mathrm{Pr} 6}\right\}_{2}(2)\left(150 \mathrm{MHz}, \mathrm{C}_{6} \mathrm{D}_{6}, 298 \mathrm{~K}\right)$ 


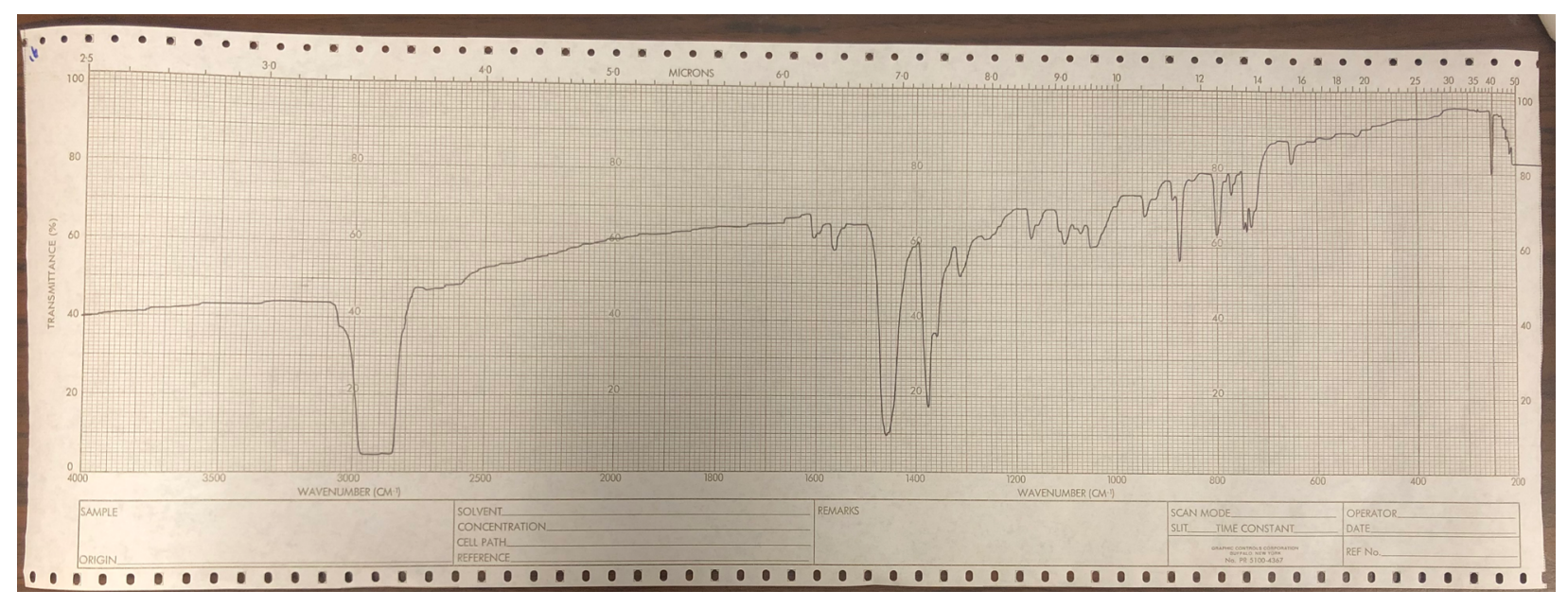

Figure S7. Infrared spectrum as a Nujol mull of $\left\{\mathrm{CuSAr}^{\mathrm{iPr} 6}\right\}_{2}(2)$ at $25^{\circ} \mathrm{C}$

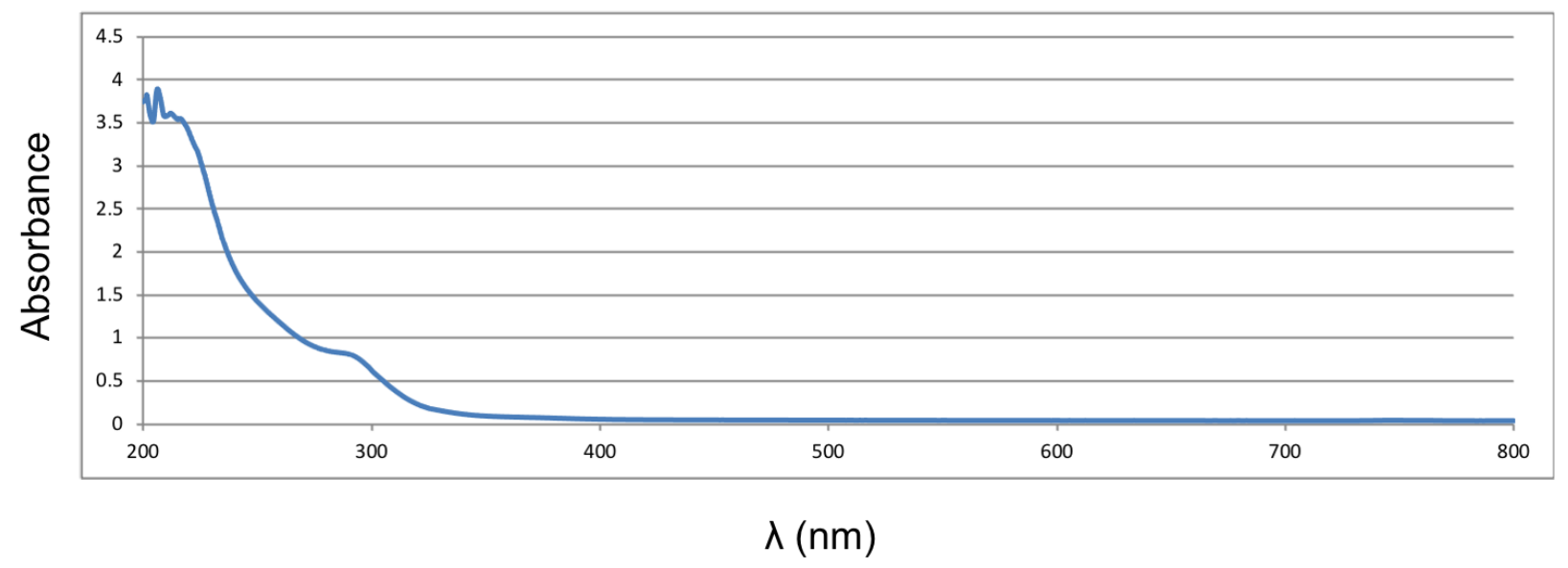

Figure S8. UV-Vis spectrum of $\left\{\mathrm{CuSAr}^{\mathrm{iPr} 6}\right\}_{2}(2)$ at $25^{\circ} \mathrm{C}(465 \mu \mathrm{M}$ in hexanes $)$ 


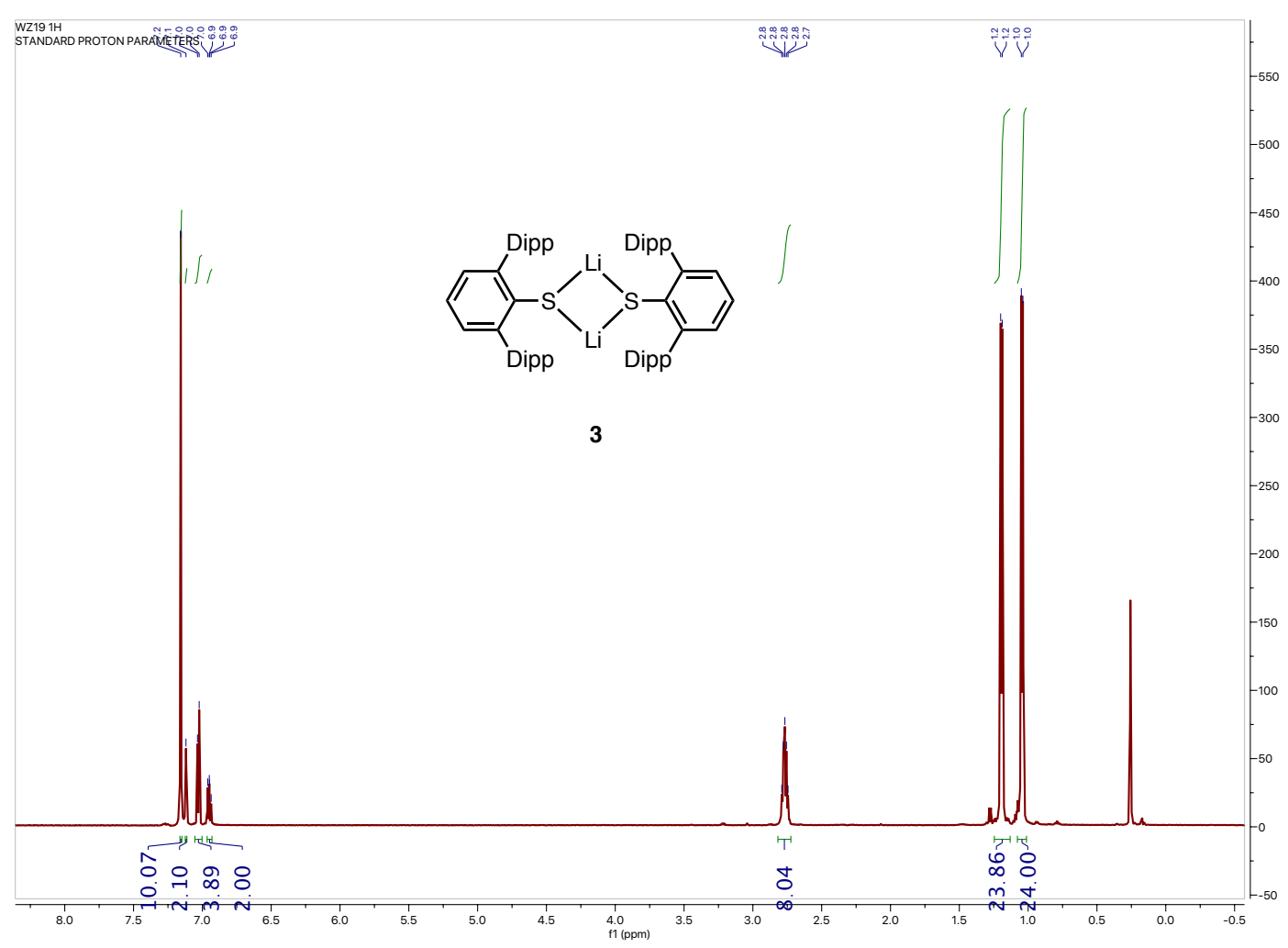

Figure S9. ${ }^{1} \mathrm{H}$ NMR spectrum of $\left\{\operatorname{LiSAr}^{\mathrm{iPr} 4}\right\}_{2}(3)\left(600 \mathrm{MHz}, \mathrm{C}_{6} \mathrm{D}_{6}, 298 \mathrm{~K}\right)$

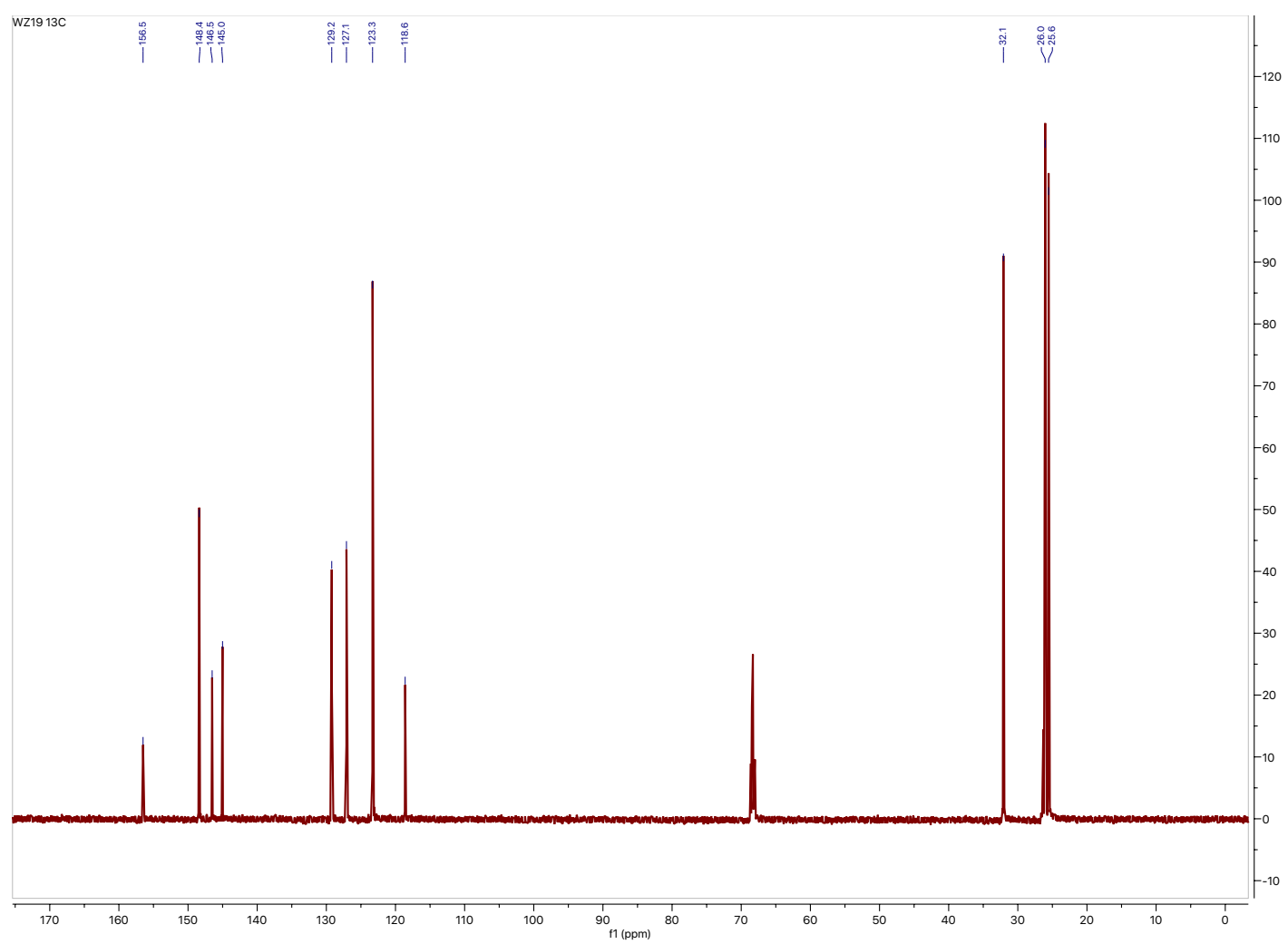

Figure $\mathrm{S} 10 .{ }^{13} \mathrm{C}\left\{{ }^{1} \mathrm{H}\right\}$ spectrum of $\left\{\mathrm{LiSAr}^{\mathrm{iPr} 4}\right\}_{2}(3)\left(150 \mathrm{MHz}, \mathrm{C}_{6} \mathrm{D}_{6}, 298 \mathrm{~K}\right)$ 


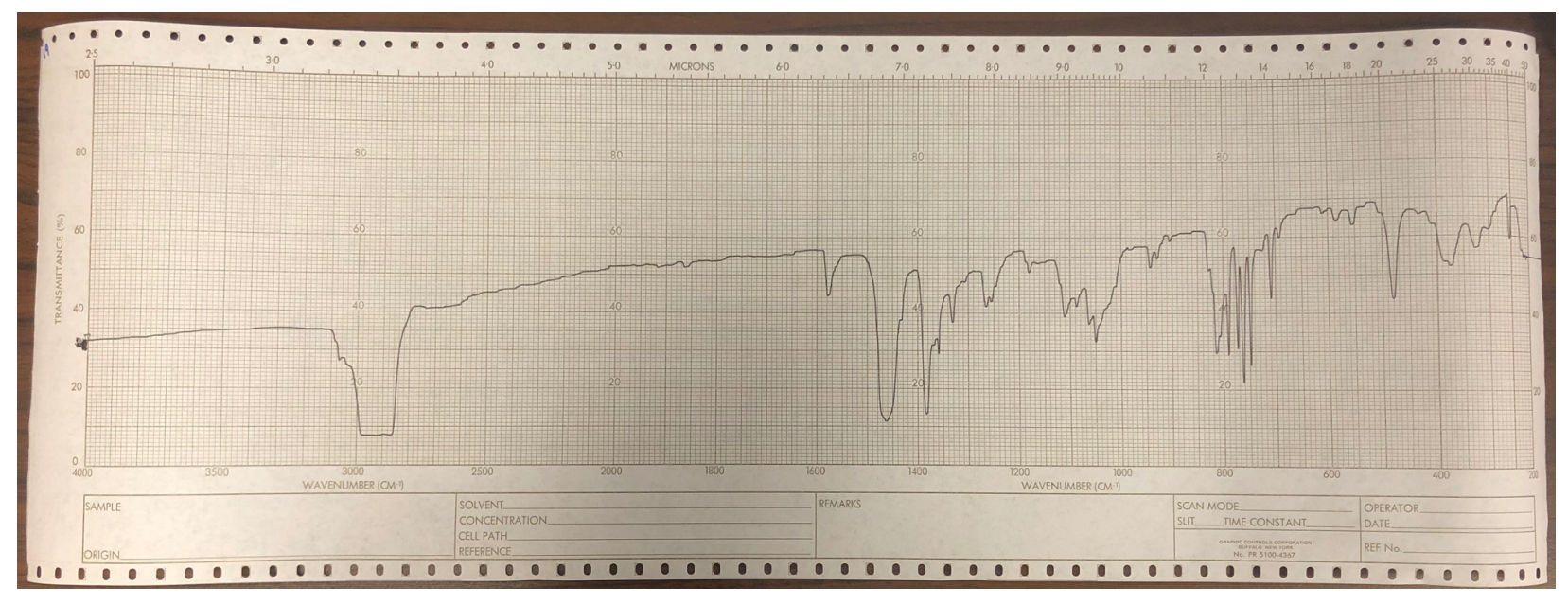

Figure S11. Infrared spectrum as a Nujol mull of $\left\{\operatorname{LiSAr}^{\mathrm{iPr} 4}\right\}_{2}(\mathbf{3})$ at $25^{\circ} \mathrm{C}$

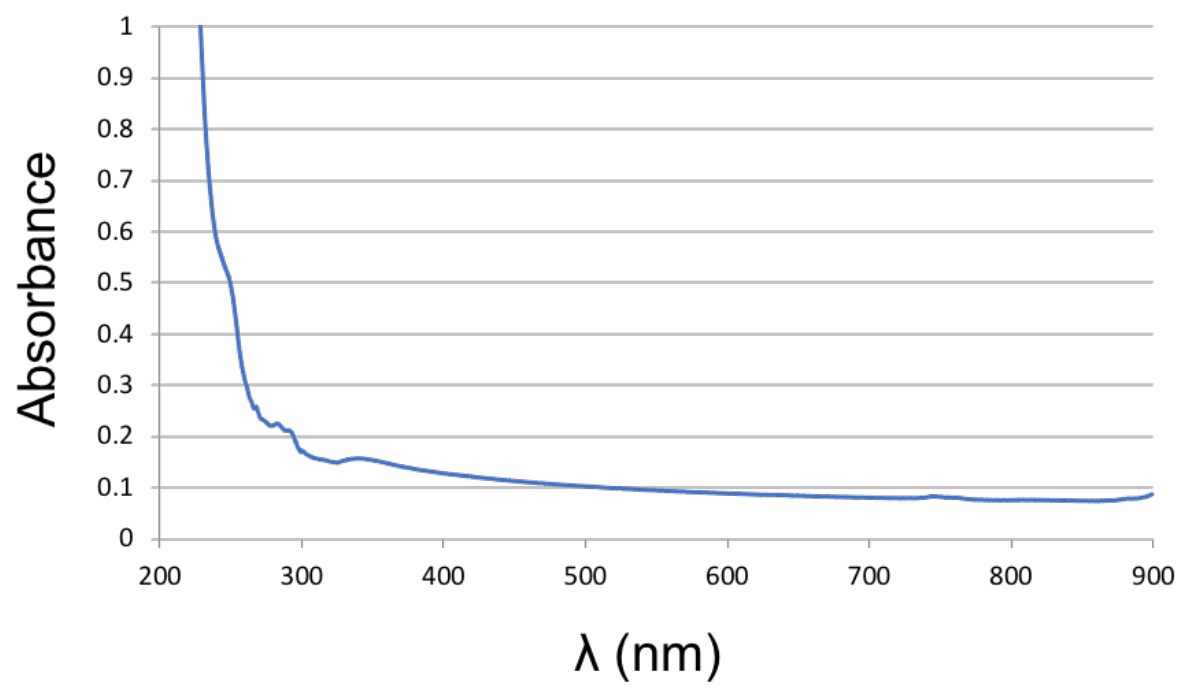

Figure S12. UV-Vis spectrum of $\left\{\operatorname{LiSAr}^{\mathrm{iPr} 4}\right\}_{2}(3)$ at $25^{\circ} \mathrm{C}(140 \mu \mathrm{M}$ in hexanes $)$ 
Table S1. Crystallographic data for compounds 1-3

\begin{tabular}{|c|c|c|c|}
\hline & 1 & 2 & 3 \\
\hline formula & $\mathrm{C}_{60} \mathrm{H}_{74} \mathrm{Cu}_{2} \mathrm{~S}_{2}$ & $\mathrm{C}_{72} \mathrm{H}_{98} \mathrm{Cu}_{2} \mathrm{~S}_{2}$ & $\mathrm{C}_{60} \mathrm{H}_{74} \mathrm{Li}_{2} \mathrm{~S}_{2}$ \\
\hline fw & 984.4 & 1152.6 & 872.6 \\
\hline color & colorless & colorless & colorless \\
\hline cyst syst & monoclinic & monoclinic & monoclinic \\
\hline space group & $\mathrm{P} 2{ }_{1} / \mathrm{n}$ & $\mathrm{P} 2{ }_{1} / \mathrm{c}$ & $\mathrm{P} 2{ }_{1} / \mathrm{n}$ \\
\hline $\mathrm{a}, \AA$ & $10.9768(7)$ & $16.4259(15)$ & $10.8353(12)$ \\
\hline $\mathrm{b}, \AA$ & $20.7437(11)$ & $16.7574(16)$ & $20.4108(17)$ \\
\hline $\mathrm{c}, \AA$ & $11.4158(6)$ & $23.022(2)$ & $11.7325(7)$ \\
\hline$\alpha, \operatorname{deg}$ & 90 & 90 & 90 \\
\hline$\beta, \operatorname{deg}$ & $90.662(5)$ & $98.527(2)$ & $91.791(12)$ \\
\hline$\gamma, \operatorname{deg}$ & 90 & 90 & 90 \\
\hline $\mathrm{V}, \AA^{3}$ & $2599(3)$ & $6266.9(10)$ & $2593.5(4)$ \\
\hline $\mathrm{Z}$ & 2 & 4 & 2 \\
\hline Density (calculated) & $1.260 \mathrm{Mg} / \mathrm{m}^{3}$ & $1.224 \mathrm{Mg} / \mathrm{m}^{3}$ & $1.118 \mathrm{Mg} / \mathrm{m}^{3}$ \\
\hline Absorption coefficient & $2.025 \mathrm{~mm}^{-1}$ & $0.786 \mathrm{~mm}^{-1}$ & $1.187 \mathrm{~mm}^{-1}$ \\
\hline $\mathrm{F}(000)$ & 1048 & 2480 & 944 \\
\hline Crystal size & $0.281 \times 0.200 \times 0.193 \mathrm{~mm}^{3}$ & $0.524 \times 0.199 \times 0.156 \mathrm{~mm}^{3}$ & $0.336 \times 0.251 \times 0.234 \mathrm{~mm}^{3}$ \\
\hline Crystal color and habit & Colorless Block & Colorless Plate & Colorless Plate \\
\hline $\begin{array}{l}\text { Theta range for data } \\
\text { collection }\end{array}$ & 4.262 to $69.395^{\circ}$ & 2.027 to $27.523^{\circ}$ & 4.332 to $68.585^{\circ}$ \\
\hline Index ranges & $\begin{array}{l}-13<=\mathrm{h}<=13 \\
-24<=\mathrm{k}<=24 \\
-13<=\mathrm{l}<=13\end{array}$ & $\begin{array}{l}-21<=\mathrm{h}<=21, \\
-21<=\mathrm{k}<=21, \\
-29<=\mathrm{l}<=29\end{array}$ & $\begin{array}{l}-12<=\mathrm{h}<=13 \\
-24<=\mathrm{k}<=24 \\
-14<=\mathrm{l}<=14\end{array}$ \\
\hline Reflections collected & 14153 & 55697 & 14958 \\
\hline Independent reflections & $\begin{array}{l}4776 \\
{[R(\text { int })=0.0159]}\end{array}$ & $\begin{array}{l}14423 \\
{[R(\text { int })=0.0308]}\end{array}$ & $\begin{array}{l}4603 \\
{[R(\text { int })=0.0289]}\end{array}$ \\
\hline
\end{tabular}




\begin{tabular}{|l|l|l|l|}
\hline $\begin{array}{l}\text { Data / restraints / } \\
\text { parameters }\end{array}$ & $4776 / 0 / 437$ & $14423 / 101 / 786$ & $4603 / 0 / 438$ \\
\hline Goodness-of-fit on $\mathrm{F}^{2}$ & 1.048 & 1.030 & 1.045 \\
\hline $\begin{array}{l}\text { Final R indices } \\
{[\mathrm{I}>2 \text { sigma(I) }}\end{array}$ & $\begin{array}{l}\mathrm{R} 1=0.0261, \\
\mathrm{wR} 2=0.0704\end{array}$ & $\begin{array}{l}\mathrm{R} 1=0.0355, \\
\mathrm{wR} 2=0.0963\end{array}$ & $\begin{array}{l}\mathrm{R} 1=0.0442, \\
\mathrm{wR} 2=0.1172\end{array}$ \\
\hline $\begin{array}{l}\mathrm{R} \text { indices } \\
\text { (all data) }\end{array}$ & $\begin{array}{l}\mathrm{R} 1=0.0268, \\
\mathrm{wR} 2=0.0709\end{array}$ & $\begin{array}{l}\mathrm{R} 1=0.0450, \\
\mathrm{wR} 2=0.1030\end{array}$ & $\begin{array}{l}\mathrm{R} 1=0.0508, \\
\mathrm{wR} 2=0.1207\end{array}$ \\
\hline $\begin{array}{l}\text { Largest diff. peak and } \\
\text { hole }\end{array}$ & $\begin{array}{l}0.317 \text { and } \\
-0.374 \mathrm{e} . \AA^{-3}\end{array}$ & $\begin{array}{l}0.579 \text { and } \\
-0.456 \mathrm{e} . \AA^{-3}\end{array}$ & $\begin{array}{l}0.310 \text { and } \\
-0.303 \mathrm{e} . \AA^{-3}\end{array}$ \\
\hline
\end{tabular}


Computational details

The structure was subjected to geometry optimization at the DFT level of theory, with the B3LYP hybrid exchange functional ${ }^{\mathrm{S} 1-\mathrm{S} 4}$ using Ahlrichs polarized basis set def2-TZVP ${ }^{\mathrm{S} 5}$ basis set together with the RIJCOSX approximation. ${ }^{\text {s6 }}$ In addition, dispersion correction with Becke-Johnson damping $(\mathrm{D} 3 \mathrm{BJ})^{\mathrm{s7}-\mathrm{S} 8}$ was used. The resolution of identity approximation was employed with auxiliary basis set def2-TZVP/C $\mathrm{C}^{\mathrm{S} 9-\mathrm{S} 10}$ in order to speed up the calculations. The UV-vis absorption spectra of optimized geometry were calculated with time dependent (TD) DFT method. All calculations were carried out using the ORCA 4.2.1 quantum chemistry package. ${ }^{\text {s11 }}$

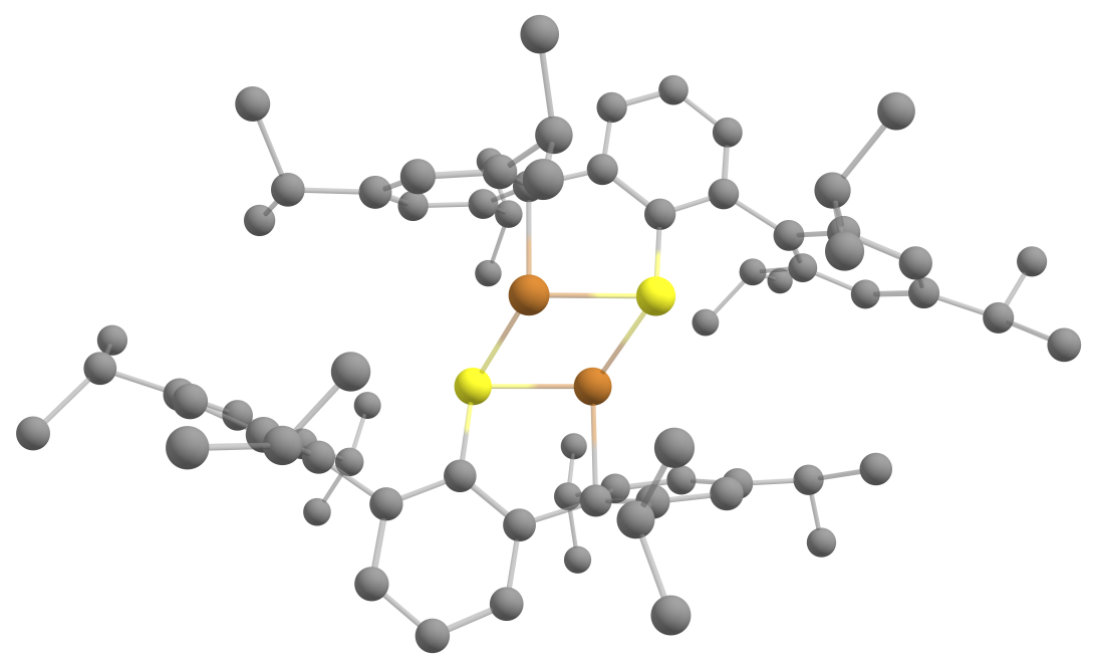

Table S2. Calculated dispersion energy of complex 2.

\begin{tabular}{|l|l|l|l|}
\hline B3LYP+def2-TZVP & B3LYP-D3BJ & B3LYP & Experimental \\
\hline $\mathrm{Cu} \cdots \mathrm{Cu}(\AA)$ & 2.417 & 2.415 & 2.4145 \\
\hline $\mathrm{Cu}(1)-\mathrm{S}(2)(\AA)$ & 2.282 & 2.418 & 2.2844 \\
\hline $\mathrm{Cu}(1)-\mathrm{S}(1)(\AA)$ & 2.323 & 2.350 & 2.3227 \\
\hline
\end{tabular}




\begin{tabular}{|c|c|c|c|}
\hline $\mathrm{Cu}(2)-\mathrm{S}(1)(\AA)$ & 2.280 & 2.417 & 2.2844 \\
\hline $\mathrm{Cu}(2)-\mathrm{S}(2)(\AA)$ & 2.322 & 2.351 & 2.3227 \\
\hline $\mathrm{Cu}(2)-\mathrm{S}(2)-\mathrm{C}(37)\left(^{\circ}\right)$ & 96.9 & 98.6 & 97.1 \\
\hline $\mathrm{Cu}(1)-\mathrm{S}(1)-\mathrm{C}(1)\left(^{\circ}\right)$ & 96.9 & 98.7 & 97.1 \\
\hline $\mathrm{S}(2)-\mathrm{Cu}(2)-\mathrm{S}(1)\left(^{\circ}\right)$ & 116.7 & 119.1 & 116.8 \\
\hline $\mathrm{S}(2)-\mathrm{Cu}(1)-\mathrm{S}(1)\left(^{\circ}\right)$ & 116.6 & 119.1 & 116.8 \\
\hline \multicolumn{4}{|c|}{$\begin{array}{c}\left(\mathrm{Ar}{ }^{\mathrm{iPr} 6} \mathrm{SCu}\right)_{2}(0,1) \rightarrow 2 \mathrm{Ar}^{\mathrm{iPr} 6} \mathrm{SCu}(0,1) \\
\mathrm{BDE}=2 \mathrm{E}\left(\mathrm{Ar}{ }^{\mathrm{PPr} 6} \mathrm{SCu}\right)-\mathrm{E}\left\{\left(\mathrm{Ar}^{\mathrm{iPr} 6} \mathrm{SCu}\right)_{2}\right\}\end{array}$} \\
\hline $\mathrm{BDE} / \mathrm{E}_{\text {disp }}(\mathrm{kcal} / \mathrm{mol})$ & 50.4 & $23.7 / 26.7$ & \\
\hline
\end{tabular}


References:

S1. Becke, A. D. A new Xixing of Hartree-Fock and local density-Functional Theories. J. Chem. Phys., 1993, 98, 1372-1377.

S2. Lee, C.; Yang, W.; Parr, R. G. Development of the Colle-Salvetti Correlation-Energy Formula into a Functional of the Clectron Density. Phys. Rev. B, 1988, 37, 785-789.

S3. Vosko, S. H.; Wilk, L.; Nusair, M., Accurate Spin-Dependent Electron Liquid Correlation Energies for Local Spin Density Calculations: A Critical Analysis. Can. J. Phys. 1980, 58, 12001211.

S4. Stephens, P. J.; Devlin, F. J.; Chabalowski, C. F.; Frisch, M. J. Ab Initio Calculation of Vibrational Absorption and Circular Dichroism Spectra Using Density Functional Force Fields. $J$. Phys. Chem., 1994, 98, 11623-11627.

S5. Weigend, F.; Ahlrichs, R. Balanced Basis Sets of Split Valence, Triple Zeta Valence and Quadruple Zeta Valence Quality for H to Rn: Design and Assessment of Accuracy. PCCP 2005, 7, 3297-3305.

S6. Neese, F.; Wennmohs, F.; Hansen, A.; Becker, U. Efficient, Approximate and Parallel Hartree-Fock and Hybrid DFT Calculations. A 'Chain-of-Spheres' Algorithm for the HartreeFock Exchange. Chem. Phys., 2009, 356, 98-109.

S7. Grimme, S.; Antony, J.; Ehrlich, S.; Krieg, H. A Consistent and Accurate Ab Initio Parametrization of Density Functional Dispersion Correction (DFT-D) for the 94 Elements H-Pu. J. Chem. Phys., 2010, 132, 154104/1-154104/19.

S8. Grimme, S.; Ehrlich, S.; Goerigk, L. Effect of the Damping Function in Dispersion Corrected Density Functional Theory. J. Comput. Chem. 2011, 32, 1456-1465.

S9. Weigend, F., Accurate Coulomb-Fitting Basis Sets for H to Rn. PCCP 2006, 8, 1057-1065. 
S10. Hellweg, A.; Hättig, C.; Höfener, S.; Klopper, W. Optimized Accurate Auxiliary Basis Sets for RI-MP2 and RI-CC2 Calculations for the Atoms Rb to Rn. Theor. Chem. Acc. 2007, 117, 587597.

S11. Neese, F.; the ORCA Program System. WIREs Computational Molecular Science, 2012, 2, 73-78. 